PAX6 gene dosage effect in a family with congential cataracts, aniridia, anophthalmia and central nervous system defects

T. Glaser, L. Jepeal, J. G. Edwards, S. R. Young, J. Favor \& R. L. Maas

Nature Genetics 7, 463-471

An old version of Fig. 4 was inadvertently printed. The mutation denoted $\mathrm{TGA}^{352}$ is actually $\mathrm{TGA}^{353}$, as correctly referred to elsewhere in the legend and text.

\section{Loss of imprinting of IGF2 is linked to reduced expression and abnormal methylation of $H 19$ in Wilms' tumour}

Marja J. C. Steenman, Shirley Rainier, Craig J. Dobry, Paul Grundy, Isabelle L. Horon \& Andrew P. Feinberg

Nature Genetics 7, 433-439

In Table 1, heading WT's with LOI, third row (NK), the last five digits ( 0 ) should be in the appropriate columns $1,2,3,4$ and 5.

The thin-lined boxes in Table 2 were left out. The revised Table 2 is printed below.
Studying human mutations by sperm typing: instability of CAG trinucleotide repeats in the human androgen receptor gene

Lin Zhang, Esther P. Leeflang, Jian Yu \& Norman Arnheim

Nature Genetics 7, 531-535

Figures 2 and 3 were inadvertantly transposed in this paper; the legends were correct, as published. Also, in the last row (Total) in Table 2, the last three digits (1) on the right hand side should be in the appropriate columns 3,5 and 6 .

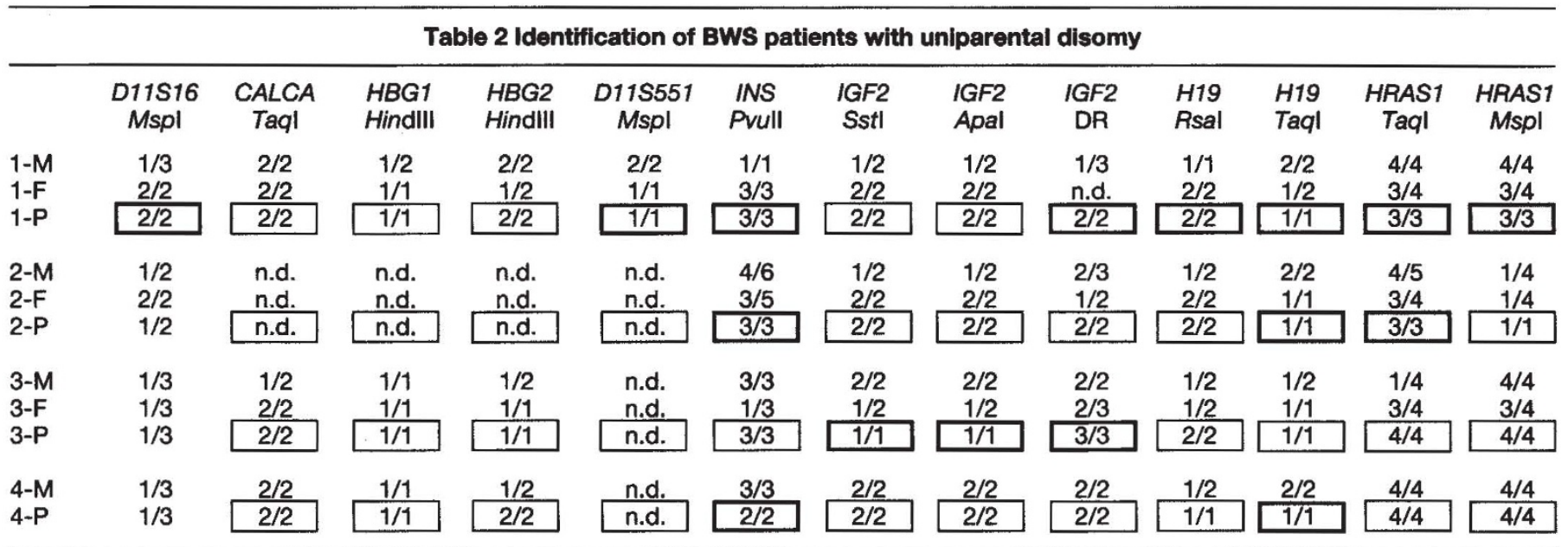

Genotypes in thick-lined boxes are those for which the patients conclusively showed paternal uniparental disomy. Genotypes for which the patients were not informative are indicated by thin-lined boxes. Alleles are numbered according to decreasing size. $M$, mother; $F$, father; $P$, patient; n.d., not determined. 